\title{
Influence of Ammonium Hydrogen Carbonate to Metal Ions Molar Ratio on Co-precipitated Nanopowders for TGG Transparent Ceramics
}

\author{
LI Xiao-Ying ${ }^{1,2}$, LIU Qiang ${ }^{2}$, HU Ze-Wang ${ }^{1,3}$, JIANG Nan $^{1,3}$, SHI Yun ${ }^{1}$, LI Jiang ${ }^{1,3}$
}

(1. Key Laboratory of Transparent Opto-Functional Inorganic Materials, Shanghai Institute of Ceramics, Chinese Academy of Sciences, Shanghai 200050, China; 2. School of Material Science and Engineering, Jiangsu University, Zhenjiang 212013, China; 3. Center of Materials Science and Optoelectronics Engineering, University of Chinese Academy of Sciences, Beijing 100049, China)

\begin{abstract}
Terbium gallium garnet (TGG) ceramics were successfully fabricated by air sintering at $1500{ }^{\circ} \mathrm{C}$ for $3 \mathrm{~h}$ combined with HIP post-treating at $1550{ }^{\circ} \mathrm{C}$ for $3 \mathrm{~h}$ under $150 \mathrm{MPa}$ argon gas, where the TGG powders were synthesized by the co-precipitation method employing ammonium hydrogen carbonate (AHC) as precipitant. The influences of ammonium hydrogen carbonate to metal ions molar ratio ( $R$ value) on phase composition and morphology of the resultant powders as well as optical transmittance and Verdet constant of the TGG ceramics were investigated systematically. The precursors with $R=3.6,4.0$ and 4.4 calcined at $1100{ }^{\circ} \mathrm{C}$ form pure TGG phase, whereas the precursor with $R=3.2$ treated at the same temperature yields the mixed phases of TGG and $\mathrm{Ga}_{2} \mathrm{O}_{3}$. The TGG powder with $R=4.0$ shows the best dispersity and homogeneity, giving rise to ceramic with the best optical quality. On the contrary, the powder with $R=4.4$ exhibits a strong agglomeration, which is closely related to the morphology of its precursor. High quality TGG transparent ceramics with the transmittance of $80.1 \%$ at $1064 \mathrm{~nm}$ can be fabricated by the nanopowder with $R=4.0$, and the Verdet constant of the TGG ceramics at $633 \mathrm{~nm}$ is rather close to that of the commercial TGG single crystals $\left(-134 \mathrm{rad} \cdot \mathrm{T}^{-1} \cdot \mathrm{m}^{-1}\right)$.
\end{abstract}

Key words: Faraday material; TGG ceramics; co-precipitation method; $\mathrm{AHC} / \mathrm{M}^{3+}$ molar ratio

Faraday rotator is one of the key optics for the isolation, the polarization control and the birefringence compensation of a laser amplifier for the high energy and high average power laser driven application ${ }^{[1-3]}$. The most significant properties of the Faraday material are the high Verdet constant and good optical quality, as well as a high thermal conductivity and superior size scalability for handling thermal effects ${ }^{[4-7]}$. To date, Tb-doped phosphate and silicate glasses are common Faraday material used in large aperture laser systems because of the superior size scalability ${ }^{[8-9]}$. However, amorphous glasses cannot meet the demand of high average power lasers due to its low thermal conductivity ${ }^{[10-11]}$. Terbium gallium garnet is considered as a promising magneto-optical material used in the visible and nearinfrared isolators because of its twice Verdet constant of Terbium-doped glass. Furthermore, the thermal conductivity of crystalline TGG is an order of magnitude greater than a typical glass ${ }^{[12-14]}$. The combination of the above factors makes TGG better suited for high average power applications. Unfortunately, the growth of high quality and large size TGG crystal is not an easy task for the evaporation of $\mathrm{Ga}_{2} \mathrm{O}_{3}$ from the melt during the crystal growth process, which leaves it far from application in high-average- power lasers ${ }^{[15-17]}$.

With the advancement of transparent ceramics fabrication technology, ceramics with high optical quality are usually preferred over single crystal for high power applications by virtue of its outstanding optical quality and excellent size scalability ${ }^{[18-19]}$. In general, transparent ceramics can be fabricated by two main methods. In conventional solid-state reaction between the commercial oxide, high calcination temperature and prolonged calcination time are necessary to gain the pure phase ${ }^{[20-21]}$. Furthermore, the extensive milling leads to possible contamination, thus degrading the optical quality $^{[22-23]}$. For this reason, several wet-chemical methods, such as Sol-Gel process ${ }^{[24]}$, microemulsion ${ }^{[25]}$, 
co-precipitation $^{[26-27]}$ and hydrothermal synthesis ${ }^{[28]}$, exhibit considerable advantages such as intimate mixing of the starting materials, excellent chemical homogeneity and low synthesis temperature ${ }^{[29-30]}$. Among the wet-chemical methods, co-precipitation method using ammonium hydrogen carbonate as precipitant is a promising route to synthesize TGG powders possessing the proper characteristics toward transparent ceramics. Characteristics of the co-precipitated powders depend on the nature of salts solution and precipitant as well as synthesis conditions. According to Dai, et $a l^{[31]}$ and Dulina, et $a l^{[32]}$, precipitant to metal ions molar ratio (defined as $R$ value) can influence the terminal $\mathrm{pH}$ during the precipitation process, and the terminal $\mathrm{pH}$ must be controlled strictly to optimize the chemical composition and morphology of nanopowders. However, the synthesis of TGG powders using co-precipitation method is rarely studied in detail, especially the influences of ammonium hydrogen carbonate to metal ions molar ratio on the properties of precursors and TGG powders.

In this work, TGG nanopowders were prepared by a co-precipitation route using $\mathrm{AHC}$ as the precipitant. With the aim of preparing pure TGG nanopowders beneficial to the fabrication of transparent ceramics, the role of the $R$ values on the phase composition and dispersity of the as-synthesized powders and final microstructures, optical quality and magneto-optical property of the TGG ceramics were systematically studied.

\section{Experimental}

Nanosized TGG powders, produced by a coprecipitation method, were used as starting materials for ceramic sample. Highly pure $\mathrm{Tb}$ and $\mathrm{Ga}$ nitrate solutions are prepared by dissolving appropriate amounts of $\mathrm{Tb}_{4} \mathrm{O}_{7}$ (99.99\%, Yuelong New Materials Co., Ltd., Shanghai, China) and $\mathrm{Ga}_{2} \mathrm{O}_{3}(99.995 \%$, Jining Zhongkai New Materials Co., Ltd., Shandong, China) powders in hot $\mathrm{HNO}_{3} / \mathrm{H}_{2} \mathrm{O}$. Then, the metal nitrates were mixed together to form a homogeneous solution according to the stoichiometric ratio of $\mathrm{Tb}_{3} \mathrm{Ga}_{5} \mathrm{O}_{12}$ and the $\mathrm{Ga}^{3+}$ concentration was set to $0.3 \mathrm{~mol} / \mathrm{L}$. Precipitant solution with a concentration of $1.5 \mathrm{~mol} / \mathrm{L}$ was obtained by dissolving ammonium hydrogen carbonate (Analytical grade, Aladdin) in deionized water. Ammonium sulfate (99.0\%, Sinopharm Chemical Reagent Co., Ltd.) was added into the precipitant solution as the dispersant. The precursor precipitate was performed by the reverse- strike method at room temperature. The molar ratio $R$ was chosen as 3.2, 3.6, 4.0 and 4.4, respectively. The white precipitate formed, and the reaction mixture was washed four times with deionized water and rinsed twice with absolute ethanol. Then, the precursor was dried at $70{ }^{\circ} \mathrm{C}$ for $48 \mathrm{~h}$. After that, the dried precursor was sieved through a $75 \mu \mathrm{m}$ screen and then calcined at $1100{ }^{\circ} \mathrm{C}$ for $4 \mathrm{~h}$. Finally, the powders were drypressed to a 20 -mm-diameter pellet at $20 \mathrm{MPa}$, then further cold isostatically pressed (CIP) under $250 \mathrm{MPa}$. The pellets were sintered at $1500{ }^{\circ} \mathrm{C}$ for $3 \mathrm{~h}$ in muffle furnace followed by hot isostatic pressing (HIP) post-treated at $1550{ }^{\circ} \mathrm{C}$ for $3 \mathrm{~h}$ under $150 \mathrm{MPa}$ in $\mathrm{Ar}$ atmosphere. The specimens were mirror-polished on both surfaces into 1.2-mm-thickness for further test.

Phase identification of the as-synthesized powders was performed via $\mathrm{X}$-ray diffraction (XRD) analysis using a Diffractometer (XRD, Mode-1 D/max2200PC, Rigaku, Japan). Specific surface area analysis was carried out by Norcross ASAP 2010 micromeritics at $77 \mathrm{~K}$, using $\mathrm{N}_{2}$ as the absorbate gas. The compositions of the precursors were examined by the Fourier transform infrared spectrometer (FT-IR, Bruker VERTEX 70 spectrophotometer, Ettlingen, Germany). The morphologies of powders and thermally-etched surfaces of the ceramic sample were submitted to FESEM characterization (S-8220, Hitachi, Japan). Grain size of the sintered sample was determined by image analysis, carried out on several micrographs acquired by FESEM and using the linear intercept method. The in-line transmittance of the specimens was measured over the wavelength region from $300 \mathrm{~nm}$ to $1800 \mathrm{~nm}$ using a spectrometer (Model Cray-5000 UV-VIS-NIR Spectrophotometer, Varian, CA, USA). The Verdet constant of the ceramics at $633 \mathrm{~nm}$ was measured using an instrument consisting of a He-Ne laser, two polarizers, and an electromagnet at room temperature.

\section{Results and discussion}

The FT-IR spectra of precursors synthesized with different $R$ values are shown in Fig. 1. It can be seen that the positions of the main absorption peaks are almost the same in the FT-IR spectra with $R=3.2-4.4$, which indicates that the molar ratio $\mathrm{R}$ has little impact on the chemical compositions of the precursors. The FT-IR spectra of precursors exhibit broad absorption bands at $3400 \mathrm{~cm}^{-1}$ corresponding to the stretching vibrations of $\mathrm{O}-\mathrm{H}$ bond ${ }^{[33]}$. The weaker absorption 


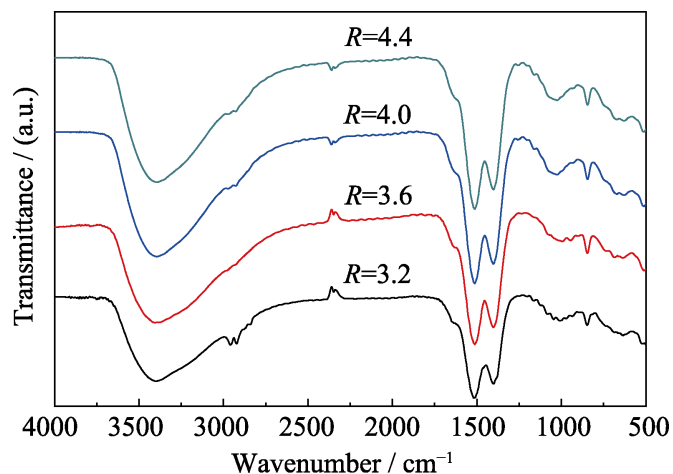

Fig. 1 FT-IR spectra of the precursors synthesized with different $R$ values

peak at $1630 \mathrm{~cm}^{-1}$ can be attributed to $\mathrm{H}-\mathrm{O}-\mathrm{H}$ bending mode of molecular water. The peak at about $1520 \mathrm{~cm}^{-1}$ results from the bond-stretching of $\mathrm{NH}_{4}{ }^{+}$. The stretching vibrations of $\mathrm{CO}_{3}{ }^{2-}$ appear as absorption peaks at $1416 \mathrm{~cm}^{-1}$, whereas the nonplanar bending $\mathrm{CO}_{3}{ }^{2-}$ vibrations are observed at $841 \mathrm{~cm}^{-1}$, indicating the presence of carbonate group in the precursors. The weak peak at $2350 \mathrm{~cm}^{-1}$ is attributed to the asymmetrical stretch of $\mathrm{CO}_{2}$ absorbed in air ${ }^{[34]}$. The abnormal absorption peaks at $2350 \mathrm{~cm}^{-1}$ of the precursors with $R=3.2$ and 3.6 are caused by baseline scan and background subtraction, which is independent of the precursors with different $R$ values.

Fig. 2 shows the FESEM micrographs of the precursors synthesized with different $\mathrm{R}$ values. It can be seen that the precursors with $R=3.2$ and 3.6 are composed of sub-micrometer sized near-spherical shaped particles. Additionally, the precursors with $R=3.2$ and 3.6 are loosely agglomerated and characterized by a high homogeneity. For the precursor with $R=4.0$, the needlelike shaped particles occur and the slight agglomeration can be observed. However, with the increase of $R$ value to 4.4, large-sized aggregate with
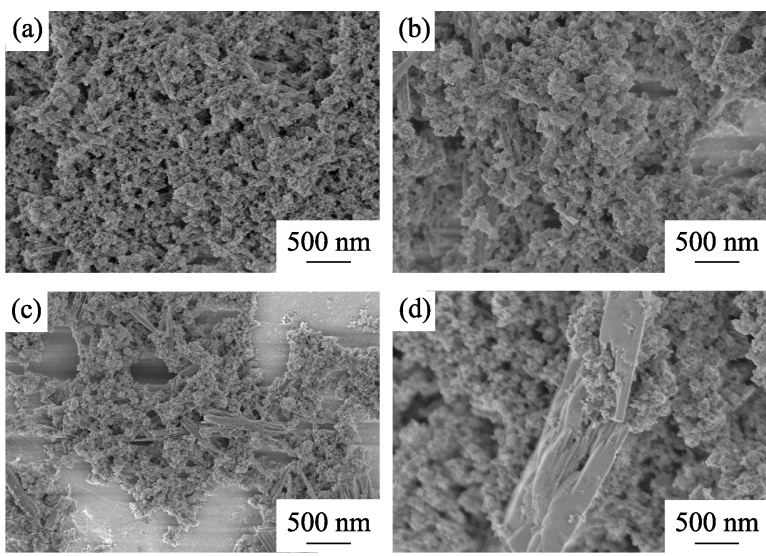

Fig. 2 FESEM micrographs of the precursors synthesized with different $R$ values

(a) $R=3.2$; (b) $R=3.6$; (c) $R=4.0$; (d) $R=4.4$ nubby morphology occurs, which is probably due to the relatively higher $\mathrm{pH}$ resulting in the enhancement of agglomeration between particles ${ }^{[35]}$.

Fig. 3 shows the XRD results of the calcined powders with different $R$ values. The results indicate that the peaks of the powders with different $R$ values match well with the standard diffraction of TGG (JCPDS 88-0575), except for the powder with $R=3.2$. The powder with $R=3.2$ shows a feeble trace of peak consisted with $\mathrm{Ga}_{2} \mathrm{O}_{3}$. It might be due to the partial $\mathrm{Ga}^{3+}$ ions begin to precipitate from the nitrate solutions at about $\mathrm{pH}=4.2$, while the production of $\mathrm{Tb}$ precipitates requires a higher $\mathrm{pH}$ value. As $R$ value decreases to 3.2, the lower terminal $\mathrm{pH}$ results in segregation of $\mathrm{Tb}$ precipitate and $\mathrm{Ga}$ precipitate. The average crystallite size $\left(D_{\mathrm{XRD}}\right)$ of the synthesized powders can be calculated from the XRD spectra using the Scherrer's formula. The average crystallite size value of TGG powders with $R=3.6,4.0$ and 4.4 are 125.9, 106.3 and $115.2 \mathrm{~nm}$, respectively.

Fig. 4 shows the dispersion state of TGG powders calcined at $1100{ }^{\circ} \mathrm{C}$ for $4 \mathrm{~h}$ with various $R$ values. For the powder with $R=3.2$, the obvious secondary phase

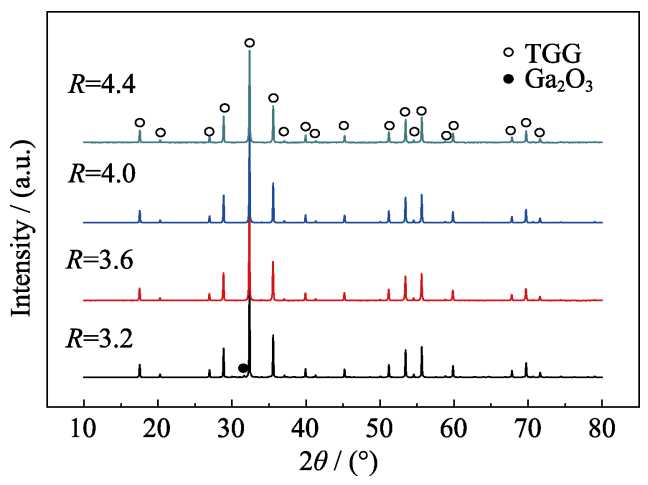

Fig. 3 XRD patterns of powders calcined at $1100{ }^{\circ} \mathrm{C}$ for $4 \mathrm{~h}$ with different $R$ values
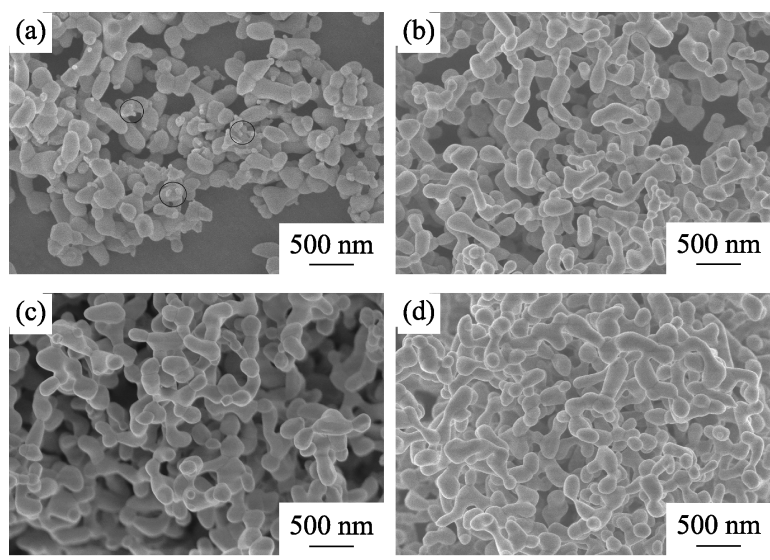

Fig. 4 FESEM micrographs of TGG powders calcined at $1100{ }^{\circ} \mathrm{C}$ for $4 \mathrm{~h}$ with different $R$ values

(a) $R=3.2$; (b) $R=3.6$; (c) $R=4.0$; (d) $R=4.4$ 
can be observed and the EDS measurement demonstrates that the composition of the secondary phase is a gallium riched phase, which is in good accordance with the XRD results. As can be seen, the synthesized TGG nanopowder with $R=3.6$ consists of loosely agglomerated dumbbell shape particles and the average particle size is about $157.4 \mathrm{~nm}$. When $R$ value is 4.0 , the powder exhibits good homogeneity and dispersity, and the morphology and average particle size of the powder are similar to the powder with $R=3.6$. The specific surface areas of TGG powder with $R=4.0$ is 5.33 and the average particle size is about $157.7 \mathrm{~nm}$. Further increase of $R$ value to 4.4 results in severe agglomeration, accompanying the increase of average particle size $(\sim 175.1 \mathrm{~nm})$, which is detrimental to the densification of green body. The morphology of TGG powder with $R=4.4$ is closely related to the agglomeration of its precursor. In general, these results highlight the key role of the $R$ value on the purity of the TGG phase, in addition, $R$ value is also used mainly for the purpose of optimizing morphology of final TGG particles.

Fig. 5 shows the photograph and the in-line transmittance of the double-polished TGG ceramics ( $1.2 \mathrm{~mm}$ thick) fabricated by the nanopowders with different $R$ values. It can be seen that the specimen with $R=3.2$ is almost opaque and the in-line transmittance is less than $5 \%$ through the entire range from the visible to $1.8 \mu \mathrm{m}$. It is owing to a mass of second phase particles in the ceramics, which is evidenced by the FESEM micrograph shown in Fig. 6(a). When $R$ value is 3.6, the TGG ceramic sample exhibits the better transparency than the sample with $R=3.2$, but the drastic decrease in the visible wavelength range can be observed. For the ceramic sample with $R=4.0$, the in-line transmittance exceeds $75 \%$ in the region of $500-1600 \mathrm{~nm}$, reaching about $80.1 \%$ at $1064 \mathrm{~nm}$, which is equal to the theoretical value. The excellent optical quality can be attributed to the high chemical purity as well as the better dispersity of TGG powder, which result in minimum optical loss arising from the absorption or scattering in the ceramic. The result shows that there is an absorption peak centered at about $487 \mathrm{~nm}$ corresponding to ${ }^{7} \mathrm{~F}_{6}{ }^{5} \mathrm{D}_{4}$ transition of $\mathrm{Tb}^{3+}$. The sample with $R=4.4$ is opaque as the result of a large number of residual pores acted as scattering centers after the HIP post-treatment.

Fig. 6 displays the SEM micrographs of the thermally etched surfaces of the TGG ceramics with different $R$ values pre-sintered in a muffle furnace at $1500{ }^{\circ} \mathrm{C}$ for
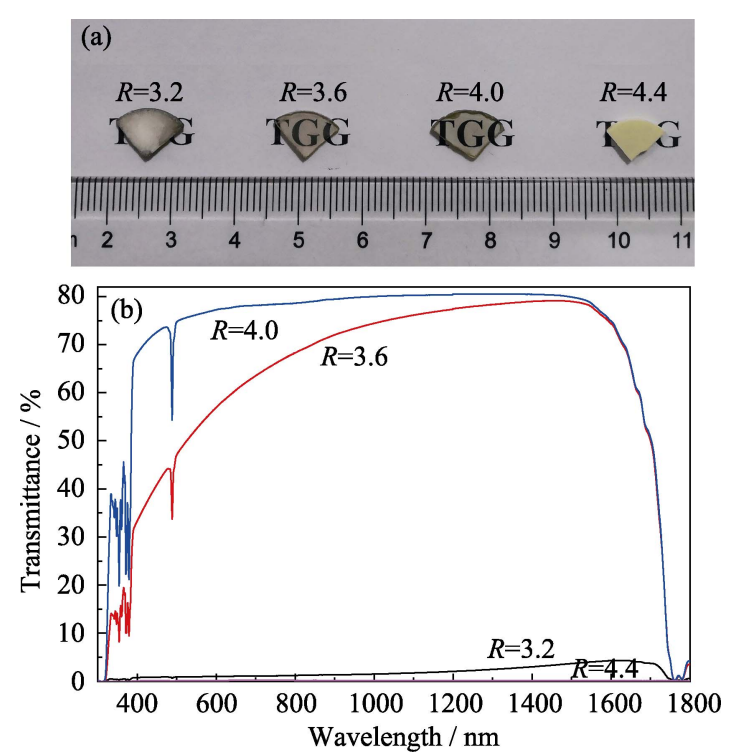

Fig. 5 (a) Photograph of TGG transparent ceramics $(1.2 \mathrm{~mm}$ thick) sintered from powders with different $R$ values and in-line transmission curves of (b) the samples pre-sintered at $1500{ }^{\circ} \mathrm{C}$ for $3 \mathrm{~h}$ in air with HIP post-treatment at $1550{ }^{\circ} \mathrm{C}$ for $3 \mathrm{~h}$ with different $R$ values
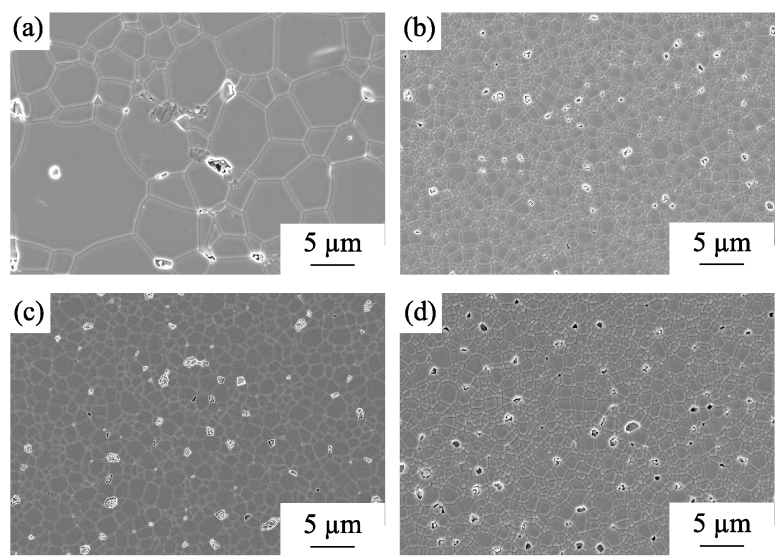

Fig. 6 FESEM micrographs of the thermally etched surfaces of TGG ceramics pre-sintered at $1500{ }^{\circ} \mathrm{C}$ for $3 \mathrm{~h}$ with different $R$ values

(a) $R=3.2$; (b) $R=3.6$; (c) $R=4.0$; (d) $R=4.4$

$3 \mathrm{~h}$. It can be noticed that the specimen with $R=3.2$ contains not only a small amount of intergranular pores and intragranular pores, but also a small amount of second phases. The EDS measurement reveals that the secondary phase is gallium oxide. The appearance of gallium oxide results from the composition segregation of the corresponding TGG powders. For the pre-sintered ceramic samples with $R=3.6,4.0$ and 4.4 , all of the pre-sintered samples are opaque because there are quite a few pores in the samples. The average grain size of TGG ceramics with $R=3.6,4.0$ and 4.4 are 1.44 , 1.63 and $1.59 \mu \mathrm{m}$, respectively. However, it can be obviously seen that the average grain size of TGG ceramics with $R=3.2$ is larger than those of other 
samples. We deduce that the superfluous $\mathrm{Ga}_{2} \mathrm{O}_{3}$ can enhance the migration rate of the grain boundary, which leads to the faster grain growth and the formation of intragranular pore.

The FESEM micrographs of the thermally etched surfaces of the HIP-treated TGG ceramics with different $R$ values are shown in Fig. 7. After the HIP-treatment, a slight grain growth occurred in all the samples, and the average grain sizes of TGG ceramics with $R=3.2$, 3.6, 4.0 and 4.4 are 9.4, 1.9, 1.8 and $2.0 \mu \mathrm{m}$, respectively. A mass of secondary $\mathrm{Ga}_{2} \mathrm{O}_{3}$ phase grains are also observed in the specimen with $R=3.2$, which act as scattering centers and lead to the low transmittance. The pores are remarkably reduced and the grain boundaries are clean without any secondary phases in the sample with $R=3.6$. However, a small number of residual pores with sub-micrometer sized are observed in the sample with $R=3.6$. The size of pores is comparable to incident wavelength, giving rise to the occurrence of Mie scattering ${ }^{[22]}$. Therefore, the transmittances of the TGG ceramics are still far from the theoretical value. For the specimen with $R=4.0$, it shows a nearly pore- free microstructure without abnormal grain growth. On the opposite, no secondary phases but large quantity of residual pores are observed in the ceramic samples with $R=4.4$, which is mainly caused by the agglomeration of the original powders.

Faraday effect leads to the rotation of polarized light and the Faraday rotation angle can be expressed by the formula:

$$
\theta=V B L
$$

For magneto-optical materials, the Faraday rotation $\theta$ is linear ratio to the Verdet constant when the length of
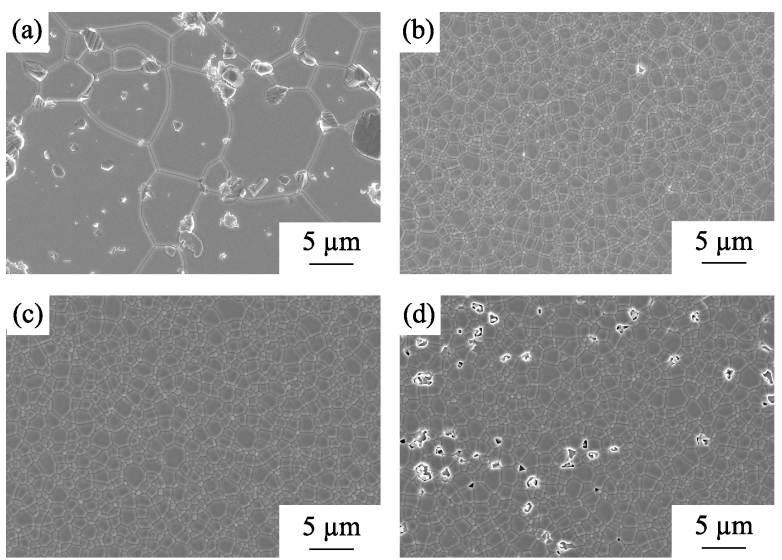

Fig. 7 SEM micrographs of the mirror-polished and thermal etched surfaces of TGG ceramics pre-sintered at $1500{ }^{\circ} \mathrm{C}$ for $3 \mathrm{~h}$ in air followed by HIP at $1550{ }^{\circ} \mathrm{C}$ for $3 \mathrm{~h}$ with different $R$ values

(a) $R=3.2$; (b) $R=3.6$; (c) $R=4.0$; (d) $R=4.4$ sample $L$ and magnetic induction intensity $B$ are fixed, so the Verdet constant is the main parameter for evaluating the magneto-optical property. In this work, the Verdet constant of TGG ceramics with $R=3.2$ and 4.4 was not measured, since the ceramics are non-transparent. The Verdet constants of the TGG ceramics with $R=3.6$ and 4.0 are -137.4 and $-136.5 \mathrm{rad} \cdot \mathrm{T}^{-1} \cdot \mathrm{m}^{-1}$, respectively, obviously indicating no significant difference between the ceramics and single crystals $\left(-134 \mathrm{rad} \cdot \mathrm{T}^{-1} \cdot \mathrm{m}^{-1}\right)$.

\section{Conclusion}

TGG precursors with different $R$ values were co-precipitated using ammonium hydrogen carbonate as the precipitant. By controlling the $R$ value to a reasonable degree, the segregation of $\mathrm{Tb}$ precipitate and $\mathrm{Ga}$ precipitate was eliminated and single phase TGG powders were obtained. The $R$ value has a significant impact on the morphology of the powders. The powder with $R=4.0$ shows the best dispersity, giving rise to denser ceramic with finer microstructures. Using these powders as raw materials, TGG transparent ceramics were successfully fabricated by air pre-sintering at $1500{ }^{\circ} \mathrm{C}$ for $3 \mathrm{~h}$ and then HIP post-treatment at $1550{ }^{\circ} \mathrm{C}$ for $3 \mathrm{~h}$. For the TGG ceramic from the powder prepared with $R=4.0$, the in-line transmittance is $80.1 \%$ at $1064 \mathrm{~nm}$. The prepared TGG magneto-optical ceramics show excellent magneto-optical properties, which is close to the TGG crystals. In future work, further efforts will concentrate on the production of high quality TGG ceramics, able to provide large aperture as well as good transparency.

\section{Reference:}

[1] YASUHARA R, SNETKOV I, STAROBOR A, et al. Terbium gallium garnet ceramic-based Faraday isolator with compensation of thermally induced depolarization for high-energy pulsed lasers with kilowatt average power. Appl. Phys. Lett., 2014, 105(24): 2175-2177.

[2] HAO D, FENG Y, TANG Y R, et al. $\mathrm{Tb}_{3} \mathrm{Al}_{2.5} \mathrm{Ga}_{2.5} \mathrm{O}_{12}$ transparent ceramic for magneto-optical application. Int. J. Appl. Ceram. Technol., 2016, 13(5): 816-820.

[3] SNETOV I L, YASUHARA R, STAROBOR A V, et al. TGG ceramics based Faraday isolator with external compensation of thermally induced depolarization. Opt. Express, 2014, 22(4): 4144-4151.

[4] YOSHIDAL H, TSUBAKIMOTO K, FUJITA H, et al. Large Diameter Ceramic TGG Faraday Rotator for High-average-power Laser Systems Depolarization. Lasers and Electro-Optics Pacific Rim. IEEE, 2016: 1-2.

[5] DAI J W, SNETKOV I L, PALASHOV O V, et al. Fabrication, microstructure and magneto-optical properties of $\mathrm{Tb}_{3} \mathrm{Al}_{5} \mathrm{O}_{12}$ transparent ceramics. Opt. Mater., 2016, 62: 205-210.

[6] YASUHARA R, FURUSE H. Thermally induced depolarization in TGG ceramics. Opt. Lett., 2013, 38(10): 1751-1753.

[7] WANG X Y, YANG L, CHEN Z, et al. Optical, magnetic suscepti- 
bilities and magneto-optical properties of neodymium doped $\mathrm{Tb}_{3} \mathrm{Ga}_{5} \mathrm{O}_{12}$ with meliorated properties for near-infrared optical isolators. J. Alloys Compd., 2015, 649: 1085-1088.

[8] QIU J R, TANAKA K, SUGIMOTO N, et al. Faraday effect in $\mathrm{Tb}^{3+}$-containing borate, fluoride and fluorophosphate glasses. $J$. Non-Cryst. Solids, 2015, 213-214: 193-198.

[9] GIESEN A, SPEISER J. Fifteen years of work on thin-disk lasers: results and scaling laws. IEEE J. Sel. Top. Quant., 2007, 13(3): 598-609.

[10] YOSHIDA H, TSUBAKIMOTO K, FUJIMOTO Y, et al. Optical properties and Faraday effect of ceramic terbium gallium garnet for a room temperature Faraday rotator. Opt. Express, 2011, 19(16): $15181-15188$.

[11] DAI J W, LI J. Promising magneto-optical ceramics for high power Faraday isolators. Scr. Mater, 2018, 155: 78-84.

[12] KAMINSKII A A, EICHLER H J, REICHE P, et al. SRS risk potential in Faraday rotator $\mathrm{Tb}_{3} \mathrm{Ga}_{5} \mathrm{O}_{12}$ crystals for high-peak power lasers. Laser Phys. Lett., 2005, 2(10): 489-492.

[13] ZHANG W J, GUO F Y, CHEN J Z, et al. Growth and characterization of $\mathrm{Tb}_{3} \mathrm{Ga}_{5-x} \mathrm{Al}_{x} \mathrm{O}_{12}$ single crystal. J. Cryst. Growth, 2007, 306(1): 195-199.

[14] KHAZANOV E A, KULAGIN O V, YOSHIDA S, et al. Investigation of self-induced depolarization of laser radiation in terbium gallium garnet. IEEE J. Quantum Electron., 2002, 35(8): 1116122.

[15] SLEZAK O, YASUHARA R, LUCIANETTI A, et al. Wavelength dependence of magneto-optic properties of terbium gallium garnet ceramics. Opt. Express, 2015, 23(10): 13641-13647.

[16] ZHUANG N F, SONG C G, GUO L W, et al. Growth of terbium gallium garnet (TGG) magneto-optic crystals by edge-defined film-fed growth method. J. Cryst. Growth, 2013, 381: 27-32.

[17] STAROBOR A, ZHELEZNOV D, PALASHOV O, et al. Study of the properties and prospects of Ce:TAG and TGG magnetooptical ceramics for optical isolators for lasers with high average power. Opt. Mater. Express, 2014, 4(10): 2127-2132.

[18] LI J, DAI J W, PAN Y B. Research progress on magneto-optical transparent ceramics. J. Inorg. Mater, 2018, 33: 1-8.

[19] DAI J W, PAN Y B, XIE T F, et al. Highly transparent $\mathrm{Tb}_{3} \mathrm{Al}_{5} \mathrm{O}_{12}$ magneto-optical ceramics sintered from co-precipitated powders with sintering aids. Opt. Mater., 2018, 78: 370-374.

[20] PALMERO P, TRAVERSO R. Co-precipitation of YAG powders for transparent materials: effect of the synthesis parameters on processing and microstructure. Materials, 2014, 7(10): 7145-7156.

[21] LI C Q, ZUO H B, ZHANG M F, et al. Fabrication of transparent YAG ceramics by traditional solid-state-reaction method. Trans. Nonferrous Met. Soc. China, 2007, 17(1): 148-153.
[22] KRELL A, KLIMKE J, HUTZLER T. Transparent compact ceramics: inherent physical issues. Opt. Mater, 2009, 31(8): 11441150.

[23] MARLOT C, BARRAUD E, GALLET S L, et al. Synthesis of YAG nanopowder by the co-precipitation method: Influence of $\mathrm{pH}$ and study of the reaction mechanisms. J. Solid State Chem., 2012, 191: 114-120.

[24] BOUKERIKA A, GUERBOUS L, BRIHI N. Ce-doped YAG phosphors prepared via Sol-Gel method: effect of some modular parameters. J. Alloys Compd., 2014, 614: 383-388.

[25] CAPONATTI E, MARTINO D C, SALADINO M L. Preparation of Nd:YAG nanopowder in a confined environment. Langmuir, 2007, 23(7): 3947-3952.

[26] LI J G, IKEGAMI T, LEE J H, et al. Co-precipitation synthesis and sintering of yttrium aluminum garnet (YAG) powders: the effect of precipitant. J. Eur. Ceram. Soc., 2000, 20(14/15): 2395-2405.

[27] PALMERO P, ESNOUF C, MONTANARO L, et al. Influence of the co-precipitation temperature on phase evolution in yttriumaluminium oxide materials. J. Eur. Ceram. Soc., 2005, 25(9): $1565-1573$.

[28] HUANG B T, MA Y Q, QIAN S B, et al. Luminescent properties of low-temperature-hydrothermally-synthesized and post-treated YAG:Ce (5\%) phosphors. Opt. Mater, 2014, 36(9): 1561-1565.

[29] LI J, CHEN F, LIU W B, et al. Co-precipitation synthesis route to yttrium aluminum garnet (YAG) transparent ceramics. J. Eur. Ceram. Soc., 2012, 32(11): 2971-2979.

[30] LI J S, SUN X D, LIU S H, et al. A homogeneous co-precipitation method to synthesize highly sinterability YAG powders for transparent ceramics. Ceram. Int., 2015, 41(2): 3283-3287.

[31] DAI J W, PAN Y B, WANG W, et al. Fabrication of $\mathrm{Tb}_{3} \mathrm{Al}_{5} \mathrm{O}_{12}$ transparent ceramics using co-precipitated nanopowders. Opt. Mater., 2017, 73: 38-44.

[32] DULINA N A, BAUMER V N, DANYLENKO M I, et al. Effects of phase and chemical composition of precursor on structural and morphological properties of $\left(\mathrm{Lu}_{0.95} \mathrm{Eu}_{0.05}\right)_{2} \mathrm{O}_{3}$ nanopowders. Ceram. Int., 2013, 39(3): 2397-2404.

[33] PUZYREV I S, IVANOV M G, KRUTIKOVA I V. Physicochemical properties of $\mathrm{Al}_{2} \mathrm{O}_{3}$ and $\mathrm{Y}_{2} \mathrm{O}_{3}$ nanopowders produced by laser synthesis and their aqueous dispersions. Russ. Chem. Bull., 2014, 63(7): 1504-1510.

[34] IVANOV M G, KYNAST U, LEZNINA M. $\mathrm{Eu}^{3+}$ doped yttrium oxide nano-luminophores from laser synthesis. J. Lumin., 2016, 169: $744-748$

[35] TONG S H, ZHAO J Y, WEN X. Preparation and properties of $\mathrm{Pr}^{3+} / \mathrm{Ce}^{3+}$ :YAG phosphors using triethanolamine as dispersant and pH regulator. Bull. Mater. Sci., 2016, 39(6): 1515-1519.

\title{
碳酸氢铵与金属阳离子摩尔比对共沉淀法合成铽镓 石榴石纳米粉体及陶瓷性能的影响
}

\author{
李晓英 ${ }^{1,2}$, 刘 强 ${ }^{2}$, 胡泽望 ${ }^{1,3}$, 姜 楠 ${ }^{1,3}$, 石 云 ${ }^{1}$, 李 江 1,3 \\ (1. 中国科学院 上海硅酸盐研究所, 透明光功能无机材料重点实验室, 上海 200050; 2. 江苏大学 材料科学与工 \\ 程学院, 镇江 212013;3. 中国科学院大学 材料科学与光电工程中心, 北京 100049)
}

摘 要: 本研究以碳酸氢铵(AHC)为沉淀剂, 采用共沉淀法制备了 $\mathrm{TGG}$ 粉体。以上述粉体为原料, 将素坏于 $1500{ }^{\circ} \mathrm{C}$ 空 气预烧 $3 \mathrm{~h}$, 然后于 $1550{ }^{\circ} \mathrm{C}, 150 \mathrm{MPa}$ 氩气气氛下 $\mathrm{HIP}$ 后处理 $3 \mathrm{~h}$ 获得 $\mathrm{TGG}$ 陶瓷。系统研究了碳酸氢铵与金属离子摩 尔比 ( $R$ 值)对合成粉体的相组成、形貌以及 TGG 陶瓷的透光率和 Verdet 常数的影响。 $R=3.6,4.0$ 和 4.4 的前驱体在 $1100{ }^{\circ} \mathrm{C}$ 煅烧形成纯相 TGG 粉体, 而 $R=3.2$ 的前驱体经相同温度㷽烧后形成了 $\mathrm{TGG}$ 和 $\mathrm{Ga}_{2} \mathrm{O}_{3}$ 的混合相粉体。 $R=4.0$ 的 TGG 粉体分散性和均匀性最好, 故制备的陶瓷光学质量最佳。 $R=4.4$ 的粉体具有较严重的团聚, 这与其前驱体形貌密 切相关。以 $R=4.0$ 的粉体为原料, 制备的 TGG 透明陶瓷在 $1064 \mathrm{~nm}$ 处的直线透过率为 $80.1 \%$ 。制备的 TGG 陶瓷在 $633 \mathrm{~nm}$ 处的 Verdet 常数和商业 TGG 单晶 $\left(-134 \mathrm{rad} \cdot \mathrm{T}^{-1} \cdot \mathrm{m}^{-1}\right)$ 几乎相等。

关 键 词: 法拉第材料; 铽镓石榴石陶瓷; 共沉淀法; 碳酸氢铵与金属阳离子摩尔比

中图分类号: TQ174 文献标识码: A 